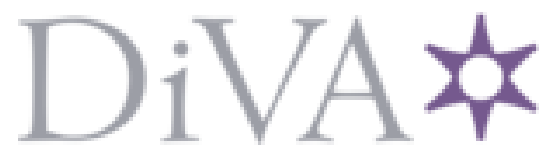

http://www.diva-portal.org

\title{
Postprint
}

This is the accepted version of a paper presented at The Fifth IFIP Conference on Sustainable Internet and ICT for Sustainability Funchal, Portugal - December 6-7, 2017.

Citation for the original published paper:

Louis Zapico, J., Hedin, B. (2017)

Energy Weight: Tangible Interface for Increasing Energy Literacy.

In: The Fifth IFIP Conference on Sustainable Internet and ICT for Sustainability, Funchal, Portugal - December 6-7, 2017 IEEE Computer Society

N.B. When citing this work, cite the original published paper.

(C)2017 IEEE

Permanent link to this version:

http://urn.kb.se/resolve?urn=urn:nbn:se:kth:diva-219814 


\section{Energy Weight: Tangible Interface for Increasing Energy Literacy}

\author{
Jorge Luis Zapico \\ Media Technology \\ Linnaeus University \\ Växjö, Sweden \\ jorgeluis.zapico@lnu.se
}

\author{
Björn Hedin \\ Department of Media Technology and Interaction Design \\ KTH Royal Institute of Technology \\ Stockholm, Sweden \\ bjornh@kth.se
}

\section{The Energy Weight Prototype}

\begin{abstract}
Increasing energy literacy has been identified as an important topic in order to help people understand their energy use and thereby enabling them to reduce their energy use. We have developed a tangible interface for helping people learn about energy by using wooden blocks as representation of several common cases of energy use. These are then placed on a digital scale connected to a computer which visualizes how many solar panels are required to power these.
\end{abstract}

Keywords-Sustainable HCI, Energy Literacy, Tangible interfaces, Technology Enhanced Learning, Work in Progress

\section{INTRODUCTION}

Studies have pointed out a general lack of a sufficient energy literacy, meaning the ability of making sense of energy information such as electricity consumption [see for instance 1,2]. In our own previous studies we could also confirm that users had not a good understanding of quantitative kilowatt hour information or of the differences in scale between different energy uses [3].

In previous studies we have explored the use of simulation and playing as a way of making sense of abstract units such as kilograms of carbon dioxide [4], and kilowatt hour [3]. In these applications, users could translate quantitative information such as $1 \mathrm{kWh}$ to more everyday activities such as charging a mobile phone. Users could also compare different everyday energy uses to each other, for example how many kilometers of driving an electric bike uses the same amount of energy as watching TV for one hour. By allowing the users to simulate and play around, these applications provide a way to anchor abstract information into more everyday experiences and in this way to improve the user's understanding when presented with such information.

This article presents an ongoing effort exploring the possibilities of taking further these ideas by using tangible interfaces [5]. The goal is to see if by embodying the information and allowing interacting with physical objects using motor skills, the learning process of understanding abstract energy information could be further improved.
The Energy Weight is a prototype that allows the user to gain a more tangible understanding of abstract energy information by being able to play and simulate with energy using physical wooden blocks representing common energy use cases. In the current prototype we use the case of solar panels for comparison. How many solar panels are required to produce the amount of energy needed for a number of common use cases? This can help the users understand the abstract value in everyday actions in a very tangible and visual way.

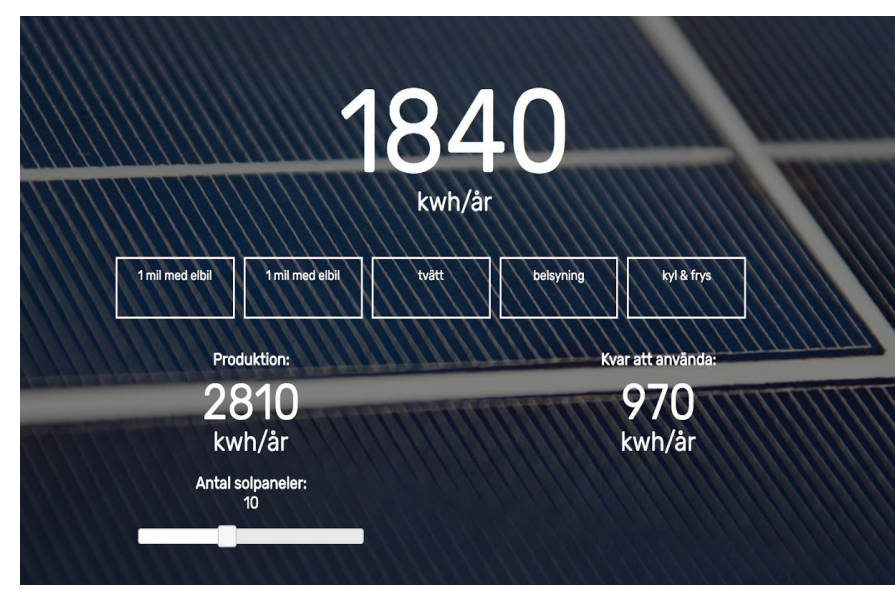

Fig. 1. The computer interface showing consumption in the top (1840 $\mathrm{kwh} /$ year), the active blocks as boxes, production in the bottom left $(2810 \mathrm{kwh} /$ year for 10 solar panels) and how much is left to use (970 $\mathrm{kwh} /$ year) .

The prototype is an application created using Node.js that connects to a bluetooth scale [6]. Wooden blocks with different weight that are put on the scale are used as the tangible interface, with their weight used as an unique id [7]. The user can select between 1 to 50 solar panels in a computer interface, and will see and approximation of how many $\mathrm{kWh}$ such a system could produce in one year. The user can then use different physical blocks that represent different energy uses (such as using a fridge during one year), which are placed on the scale to see how much that electricity is enough for. 
When the user puts one block in the tangible interface, the system uses the weight of the block to detect which action it represents, and the energy amount equivalent for that action is subtracted from the solar panels production. When the user removes one block, the electricity used will be then added back. By accumulating blocks in the interface, the user can get an idea of how much the solar panels can produce, or how many solar panels would be necessary for the intended use. By testing different blocks, the user can also gain knowledge of the differences in scale between different actions.

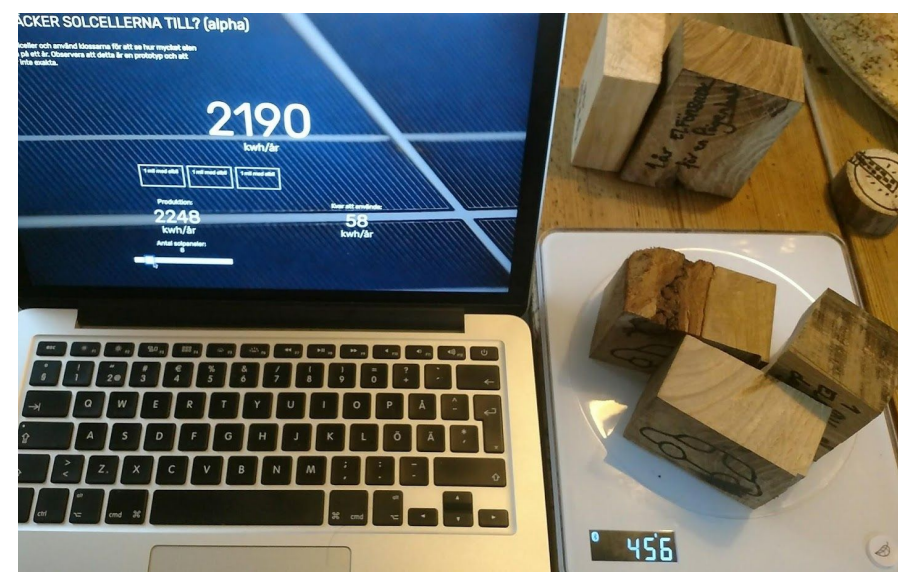

Fig. 2. The wooden blocks placed on the scale. The weight of the blocks is used to identify which blocks are placed on the scale.

At the moment the prototype has thirteen blocks made out of different wood types, with increasing weight in relation to their energy use. At the moment the weight, while representative, is not exactly proportional to the energy use.

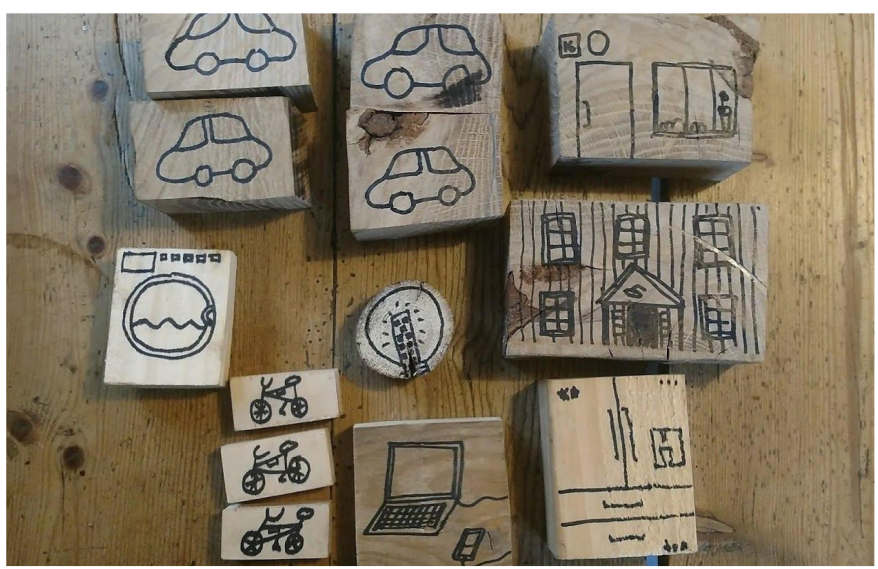

Fig. 3. The wooden blocks used to represent common cases of energy use.

The blocks are divided in three categories:

a. Transportation:

- Driving an electric car $10 \mathrm{~km}$ every day for a year (4 blocks).

- Cycling with an electric bike $10 \mathrm{~km}$ every day for a year (3 blocks)

b. Household items:
- IT and entertainment equipment for a year (calculated for two mobiles, two laptops and one television).

- Normal use of LED lightbulbs for a house during a year.

- Washing machine use during a year (200 $40^{\circ}$ cycles)

- Fridge and freeze use during a year. c. General:

- An average electricity use for an apartment (without transportation).

- An average electricity use for a house (without transportation).

\section{Discussion}

\section{Intended uses}

The Energy Weight prototype could be used in many situations. One case is classroom teaching when learning about and discussing energy. Even adults have very vague conceptions about how much energy is used by different appliances, and children are expected to have ever lower knowledge. By making the learning experience much more tangible and visual, the learning could be both more engaging and less abstract.

Another use case is trade fairs related to homes and/or energy. Especially for suppliers of solar panels such a tool could help their potential customers to better understand the possibilities and limitations of solar panels. The tool could also be used for energy advisors, such as the Swedish "Municipal Energy and Climate Advisors" who have as one of their goal to educate people about the production and use of energy. Since special hardware (the tool), software (the software) and gadgets (the blocks) are needed, the use is limited to people or organizations who can afford a modest investment (about $\$ 80$ for the scale).

\section{Technology:}

The prototype uses a bluetooth scale to identify blocks by their weight. This is not the intended use of such a scale so there are several limitations we have encountered:

- $\quad$ The weight after putting or removing a block fluctuates before reaching the correct value. This is accounted for in the code by only triggering the block recognition once the value has been stable during several readings. Increasing this stabilization time too much makes the interface seem slow, while having a short time increases the error frequency of detecting another block with an intermediate weight.

- The weight of the blocks may vary depending on the calibration of the scale. The code checks the given weight plus minus two grams to allow for this variation, but this solution implies that blocks can not have weight values too close to each other, which would limit the amount of blocks that could be made. 
Even if the code account for these mentioned problems, the need for reliability of detecting unique IDs using the scale takes a toll on the speed, which makes the interaction a bit sluggish. This could be improved in future versions.

Another technological option would be to use RFID tags in the objects as unique IDs. The interaction would need a RFID reader that can read multiple tags in a surface to see which objects are on place and detect adding and removing. This solution would increase the reliability and allow adding and removing multiple objects. It would on the other hand require an advanced RFID reader with anticollision instead of the simpler RFID readers available in for example mobile phones. Other contactless solution could be also be relevant.

\section{Design decisions:}

The prototype uses at the moment one year as the time unit. This is so to average out production, and to provide a total view of a year production and consumption. But in reality, the solar panels would produce most of the electricity during the spring and summer months (especially in countries such as Sweden) while the consumption such as driving an electric car is more evenly distributed through the year. So while the consumption and production matches, in reality there would be a surplus of energy in summer and a deficit in winter, that would even out in a year basis. The usefulness of using a whole year needs to be tested out to confirm the user's understanding of it. The existing blocks in this prototype include transportation, appliances and total household consumptions. These could be refined and would also need to be refined through user tests.

The energy use is based on quite rough calculations at this moment and they could be improved. For the prototypes purpose, some margin of error in the values is not a problem, as the main purpose is learning about the differences in scale. But as the visualization shows an exact number, the uncertainties in the data are hidden. This could be improved from a design perspective by either pointing out the inexactness of the numbers, or by making the assumptions and models behind the numbers visible.

The design of the blocks themselves can be improved. Now there is a rough relationship between the size of the blocks and the energy use. If more exact proportions were used, the visual memory of the blocks in itself would be a potential strong factor for learning. One way would be to 3D print models of each of the use cases with a weight/volume proportional to its energy use. However, since doubling the length of a 3D model increases the weight by a factor of eight, people could underestimate the weight of larger models. One possible alternative could be to use the same area for the base of all models, thereby making the height of the model directly proportional to its energy use, which would be both intuitive and create a strong visual memory of the proportions.

\section{Further work:}

Apart from improving the design, the next step would be to do user studies using the system. One user study will be to evaluate the learning effect of using this prototype, as compared to other ways of educating people in energy awareness which we have previously evaluated [3]. Using the system for trade fair demonstrations and doing a qualitative evaluation of the use by visitors to the trade fair is another use-case we will do together with the Swedish Municipal Energy- and Climate Advisors.

A system like this can also be used for other scenarios. One sustainability-related use-case we will look into is food and greenhouse gas (GHG) emissions. Instead of comparing to solar panels such a system could compare to a target ceiling of GHG emissions, and the blocks would instead represent different foodstuff.

\section{CONCLUSION}

This article presents the work in progress of a prototype showcasing the possibilities of using a tangible interface for interacting with energy information. By using a bluetooth scale and physical blocks, the users can test how much electricity is produced by solar panels and how it compares with the electricity used by different activities. By embodying the information, the learning could be both more engaging and less abstract. While there are still technology and design issues to improve, this prototype points to ways these type of technology could be used for increasing energy literacy.

\section{REFERENCES}

[1] J.E. DeWaters, S.E. Powers.w 2011. Energy literacy of secondary students in New York State (USA): A measure of knowledge, affect, and behavior. Energy Policy 39 1699-1710

[2] D. Brounen, N. Kok, J.M. Quigley. 2013. Energy literacy, awareness, and conservation behavior of residential households. Energy Economics $3842-50$

[3] B. Hedin and J. Zapico. 2017. Kilowh.at -- Increasing Energy Awareness Using an Interactive Energy Comparison Tool," in PERSUASIVE 2017, Amsterdam, The Netherlands, April 4--6, 2017, Proceedings, P. W. de Vries, H. Oinas-Kukkonen, L. Siemons, N. Beerlage-de Jong, and L. van Gemert-Pijnen, Eds. Cham: Springer International Publishing, 2017, pp. 175-185.

[4] JL Zapico Lamela, M Turpeinen, M Guath. 2011. Kilograms or cups of tea: Comparing footprints for better $\mathrm{CO} 2$ understanding. PsychNology Journal 9 (1), 43-54.

[5] Shaer, O., \& Hornecker, E. 2010. Tangible user interfaces: past, present, and future directions. Foundations and Trends in Human-Computer Interaction, 3(1-2), 1-137..

[6] Situ scale http://situscale.com/product/situ/

[7] Code available at: https://github.com/QuantifiedSelfEnergy/Energy-Weight 